\title{
Une approche pour la sélection de variables stables : application à l'encodage des diagnostics secondaires
}

\author{
Ghazar Chahbandarian ${ }^{1}$, Nathalie Bricon-Souf ${ }^{1}$, Imen Megdiche ${ }^{1}$, \\ Rémi Bastide $^{1}$, Jean-Christophe Steinbach ${ }^{2}$
}

1 IRIT, Université de Toulouse, CNRS, UPS - 118 route de Narbonne,

31062 Toulouse Cedex 9, France

Email: \{ghazar.chahbandarian,nathalie.souf, imen.megdiche, remi.bastide\}@irit.fr

2 Centre Hospitalier Intercommunal de Castres Mazamet, Department of Medical Information,

81100 Castres, France

Email: jean-christophe.steinbach@chic-cm.fr

RESUME Dans cet article, nous proposons une approche pour sélectionner des variables stables dans le contexte de prédiction des diagnostiques secondaires en partant d'une base de données médico-économique, en l'occurrence le PMSI. Les résultats de prédiction se présentent sous forme de guides pour l'activité d'encodage des diagnostiques secondaires dans les départements d'information médicale (DIM). L'approche que nous proposons dans ce papier consiste à exploiter les paradigmes ensemblistes sur les sources de données réduites et équilibrées pour déduire un ensemble stable et fiable de variables utiles à la prédiction. Cet ensemble est construit de façon très indépendante par rapport à l'échantillon de données utilisé pour l'apprentissage du modèle. La qualité des variables est déduite en fonction de la qualité de prédiction des algorithmes de ML. L'évaluation de notre approche sur les données de PMSI montre le réel intérêt de cette proposition et ouvre le débat sur l'application de ces méthodes à ces sources de données très rarement exploitées par la communauté scientifique.

ABSTRACT. In this paper, we focus on applying feature selection in the context of secondary diagnoses prediction starting from medico-economic data sources. The results of the prediction is used as guidelines for encoding secondary diagnoses which is a sensitive task in the hospitals requiring a lot of attention in order to be achieved properly. We propose a practical approach to select stable and relevant features from imbalanced datasets. The stability of features is obtained through the convergence of several FS methods to a fair number of features without being impacted by the sampled dataset. The quality of featured shall be deducted from the quality prediction of machine learning algorithms on the selected features. We evaluate the proposed approach on the PMSI database of the CHIC-CM 
40 TSI. Volume $37-n^{\circ} 1-6 / 2018$

hospital. Our results are quite interesting and opening discussions for these specific health care data supports.

KEYWORDS: Machine learning; Feature Selection; Medical informatics

DOI:10.3166/tsi37-0002 @ 2018 Lavoisier

\section{Introduction}

Dans le domaine de la santé, la quantité d'information médicale est en augmentation rapide étant donné que la maturité des systèmes d'information médicale permet de recueillir de plus en plus de données. L'utilisation primaire de ces données concerne principalement les offres de soins pour un objectif curatif. Néanmoins, il reste encore difficile de proposer une utilisation secondaire efficace pour de telles masses de données alors même qu'avec les progrès des sciences de l'information, il devient intéressant d'exploiter cette énorme quantité de données pour acquérir et inférer de nouvelles connaissances, dans des perspectives connexes aux objectifs initialement suivis lors du recueil.

Notre étude se concentre sur l'analyse d'une base de données médicale particulière utilisée en France appelée PMSI (Programme de Médicalisation des Systèmes d'Information) qui contient des informations à visée médico-économique décrivant les séjours des patients. Chaque mois, les hôpitaux transmettent une description concise et pseudo-anonymisée des différents séjours des patients aux agences nationales de santé, ce qui permet dans le cadre de la T2A (tarification à l'activité) de fournir aux hôpitaux un paiement équitable, en fonction de leur activité encodée. Un département hospitalier spécifique (le DIM, Département d'Information Médicale) est en charge de la collecte, du traitement et de la transmission des informations médicales anonymisées relatives aux patients, dans le respect du code de déontologie médicale ainsi que des règles relatives au secret médical. Cette base possède une structure bien définie, elle est disponible à différents niveaux car les données issues de chaque hôpital peuvent être agrégées à l'échelle régionale ou nationale. Les informations contenues dans cette base, même si elles peuvent être améliorées, sont très contrôlées et constituent une première approche d'informations exhaustives et fiables et ce, pour une couverture à l'échelle nationale.

La base de données PMSI contient des informations codées concernant les épisodes d'hospitalisation, y compris les informations administratives, les actes médicaux et les diagnostics. Le codage des diagnostics - sur lequel nous nous focalisons plus précisément dans cette étude - n'est pas facile car il nécessite une très bonne connaissance des dossiers médicaux et implique, pour le professionnel de santé en charge du codage, la lecture et l'analyse de toutes sortes d'informations telles que les lettres de décharge, les rapports et les images radio d'un épisode d'hospitalisation. Le professionnel de santé a ainsi comme objectif de retrouver tous les diagnostics liés au séjour, de les classer selon leur type, puis de les encoder. Deux principaux types de diagnostics peuvent être codés: un diagnostic principal (DP) se réfère à la maladie principale justifiant une hospitalisation (par exemple une 
lésion cardiaque), des diagnostics secondaires (DS) peuvent également être trouvés, ils informent alors sur d'autres maladies des patients (comme par exemple obésité ou diabète). Les codes de diagnostic, issus de classifications reconnues, sont alors enregistrés dans la base de données locale de l'hôpital.

Les diagnostics secondaires sont importants car ils traduisent la globalité des pathologies prises en charge ainsi que les complications. Au niveau économique, il est important de coder ce type de diagnostics pour l'hôpital car le classement des séjours patient en termes de sévérité prend en compte l'ensemble des diagnostics (principal et secondaires) et le budget alloué pour l'activité va dépendre de cette sévérité. Au niveau médical, le PMSI permet de mener des analyses de type santé publique et il est important d'avoir des informations les plus justes possibles. Or, les diagnostics secondaires sont souvent difficiles à recueillir, car les maladies impactées peuvent être mentionnées de façon beaucoup plus diffuse que pour le diagnostic principal qui est l'objet de l'hospitalisation. L'étude de (Potignon et al, 2010) a ainsi montré que, pour un tiers des patients dénutris ou obèses, ces diagnostics secondaires n'étaient pas correctement encodés.

L'objectif global du projet est ainsi de proposer des règles et aides pour détecter des contextes patients qui nécessiteraient une recherche attentive des diagnostics secondaires pressentis. Nous adoptons pour ce faire une méthode originale, car nous avons décidé d'étudier si l'application de méthodes d'apprentissage automatique (Machine Learning : ML), à partir uniquement des données PMSI, pourrait aider les professionnels de la santé à coder un diagnostic secondaire en connaissant le diagnostic principal. Même si les données PMSI sont assez pauvres en contenu, l'existence de ces bases de données anonymisées, exhaustives et de couverture nationale nous invitait à explorer si l'exploitation de ces informations via des méthodes de fouilles de données récentes pouvait conduire à une aide pour l'encodage des diagnostics secondaires.

Dans le cas d'études rétrospectives, l'application de méthodes d'apprentissage sur des bases de données médicales n'est pas facile, notamment parce que les données des différentes bases sont hétérogènes, non normalisées et les informations recueillies reflètent des parcours de soins extrêmement anarchiques. Les informations spécifiques à un objet d'étude ou à une pathologie cible sont très diluées dans l'ensemble des informations disponibles. On peut ainsi observer que la plupart des bases de données médicales sont déséquilibrées. Les jeux de données déséquilibrés contiennent les instances de la classe ciblée (celle que nous cherchons à prédire par exemple) qui sont généralement sous-représentées parmi les autres classes de l'ensemble de données. Cette distribution déséquilibrée des instances peut atteindre un ratio de 1:1000 dans certaines bases de données médicales. A titre d'exemple, l'insuffisance respiratoire, une pneumopathie commune, concerne au mieux $1 \%$ des épisodes d'hospitalisation contenus dans certaines bases de données médico-économiques

De plus, l'énorme quantité de variables (appelées aussi features) issues des grands nombres d'exemples complique l'analyse de ces bases de données si l'on 
souhaite mettre en œuvre des méthodes de ML. Le choix d'un processus de sélection de variables (Feature Selection: FS) précède la phase de modélisation des bases et permet l'application des techniques de ML, cette phase a un impact significatif sur les résultats de telles méthodes. La FS est en passe de devenir un sujet brûlant à l'ère des données volumineuses (Wang et al., 2016, Bolón-Canedo et al., 2015) et particulièrement dans le domaine des soins de santé où une grande variété de données peut être étudiée. La qualité des algorithmes d'apprentissage automatique est étroitement liée aux caractéristiques sélectionnées pour mettre en œuvre l'apprentissage.

Les méthodes d'échantillonnage ont un impact sur la sélection des attributs car la composition initiale du jeu de données est différente du jeu de données échantillonné. Appliquer des méthodes d'échantillonnage adaptées sur les jeux de données permet de résoudre les problèmes liés à des bases de données déséquilibrées que l'on retrouve dans les bases complexes, telles que la base PMSI sur laquelle nous nous appuyons. Parmi les méthodes d'échantillonnage, on dispose de méthodes de sur-échantillonnage pour lesquelles la composition des jeux de données est modifiée afin d'équilibrer le nombre d'exemples positifs et négatifs en générant plus d'exemples et de sous-échantillonnage pour lesquelles il s'agit de supprimer certains exemples.

Malgré le nombre assez important de méthodes de FS proposées dans la littérature, il n'existe pas de méthode qui convienne à tous les cas en raison de plusieurs problèmes tels ceux que nous venons de citer (ensembles de données déséquilibrés, rareté de certains types de données et problème de ratios caractéristiques/échantillons). Lorsque le jeu de données est déséquilibré et comporte une grande quantité de variables, les méthodes de sélection de variables (FS) traditionnelles ne parviennent pas à fournir des caractéristiques stables et pertinentes pour prédire la classe ciblée. Nous étudions ainsi cet effet.

L'idée est de sélectionner des variables stables quelle que soit la méthode d'échantillonnage utilisée pour équilibrer le jeu de données. Les questions sur lesquelles nous nous concentrons sont alors: "Quelle est la stabilité des variables lorsqu'une méthode FS est appliquée sur un jeu de données échantillonné?" et "Combien de jeux de données sont nécessaires pour sélectionner des variables stables?"

La principale motivation de ce travail est que la sélection de caractéristiques pertinentes joue un rôle crucial dans l'amélioration de la qualité de la prédiction des méthodes de ML pour ce type de source de données. De plus, comme le modèle appris servira de guide pour l'aide à la décision médicale, de tels résultats doivent être de haute qualité, la solution proposée doit offrir une bonne interprétabilité, permettant aux utilisateurs d'évaluer la validité de l'aide proposée. En réduisant le nombre de variables et en proposant des caractéristiques stables et pertinentes, nous nous attendons à produire un modèle interprétable dans lequel les praticiens pourraient avoir confiance. 
Ainsi, nous proposons et décrivons dans cet article une approche pour gérer le processus de la FS dans le contexte des données médico-économiques du PMSI en organisant le reste de cet article comme suit : dans la section 2, les données PMSI sont présentées. Dans la section 3, nous proposons un aperçu des travaux connexes. La section 4 est consacrée à l'explication de l'approche proposée et de ses résultats. Dans la section 5, nous discutons des forces et faiblesses de telles propositions. Enfin, nous concluons en introduisant plusieurs perspectives dans la section 6 .

\section{Données PMSI}

Dans ce chapitre, nous présentons certaines caractéristiques, importantes pour notre travail, de la base de données du PMSI.

La base de données PMSI a une structure commune pour des utilisations à différentes échelles : les données issues d'un hôpital local s'agrègent dans une base nationale et on peut ainsi atteindre des niveaux d'échelles locale, régionale et nationale. Notre recherche est réalisée en collaboration avec les médecins responsables de la base de données PMSI du Centre Hospitalier InterCommunal de Castres Mazamet (CHIC-CM), un hôpital départemental en France.

Le PMSI contient des rapports de sortie pseudo-anonymisés (RSA). Chaque rapport décrit un épisode d'hospitalisation à travers deux types d'informations:

- Informations administratives telles que la date d'admission, la date de sortie, le mode d'admission, le mode de sortie (transfert, décès), la durée du séjour, le sexe, l'âge. Cette catégorie regroupe les informations sur le patient et les types d'informations sur les unités médicales décrites dans (Lecornu et al. 2009).

- Des informations cliniques telles que le diagnostic principal (DP) qui motive l'épisode hospitalier, les diagnostics secondaires (DS) sur lesquels nous voulons nous concentrer et les diagnostics associés (DR). Il contient également tous les actes médicaux effectués pendant l'épisode d'hospitalisation.

Pour coder les diagnostics, une classification internationale des maladies (International Classification Disease ICD) est proposée et utilisée dans de très nombreux pays. En France, les professionnels de santé utilisent la version française de cette classification, dans sa dixième version, nommée CIM-10. Il s'agit d'une classification hiérarchique : les premiers niveaux d'organisation consistent en chapitres regroupant les mêmes maladies caractéristiques (comme le chapitre II consacré aux maladies tumorales), les catégories permettent d'affiner cette classification. Actuellement, environ 2049 catégories sont couramment utilisées pour le codage. Le dernier niveau décrit précisément chaque maladie et la CIM-10 contient 33816 codes dans lesquels les trois premiers caractères représentent les catégories de codes. Ces trois caractères permettront dans nos travaux (cf 4.1.1) d'évoquer de façon suffisamment informative une maladie. 
44 TSI. Volume $37-\mathrm{n}^{\circ} 1-6 / 2018$

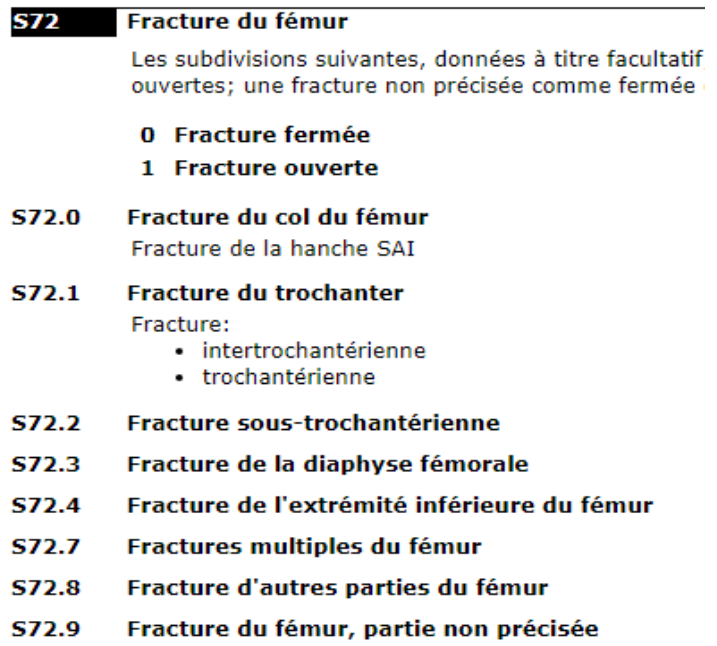

Figure 1. Exemple d'un codage (ici S72 fracture de fémur) avec la CIM-10 ( extrait de https://icd.who.int/browse10/2008/fr\#/S70-S79)

Pour coder les actes médicaux, la Classification Commune des Actes Médicaux CCAM est utilisée. C'est aussi une classification hiérarchique : les premiers niveaux sont constitués des chapitres qui organisent les procédures médicales en fonction du système médical impacté (tel que le système nerveux). Il existe environ 1700 codes d'actes médicaux standards classés en 19 chapitres. 


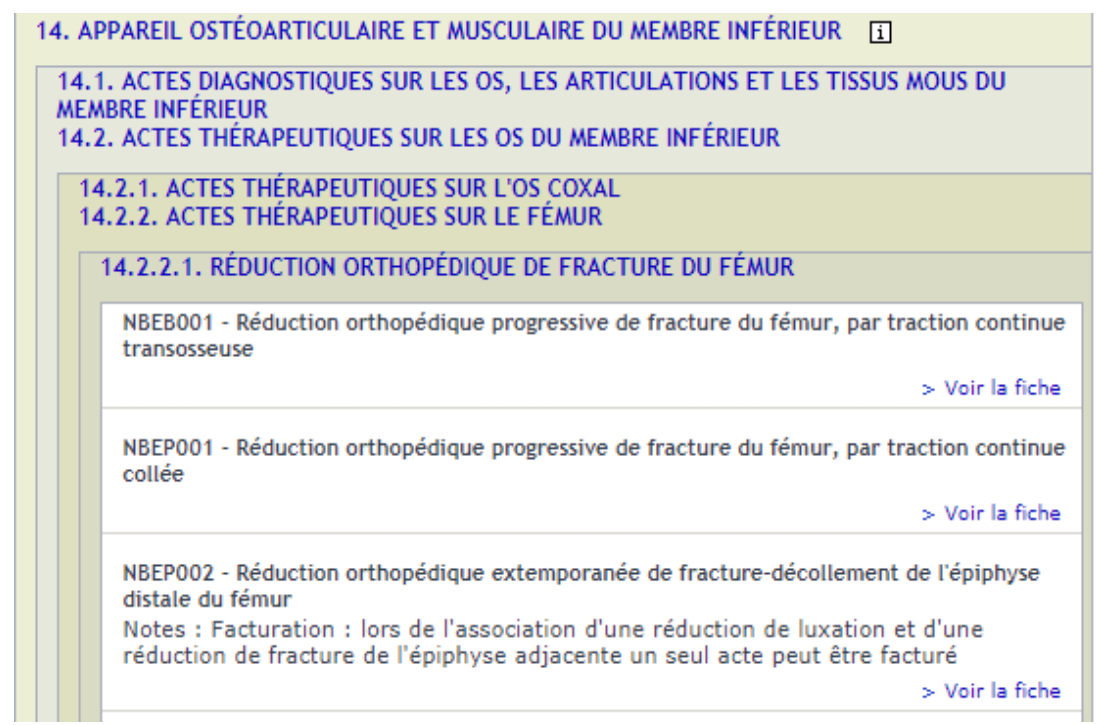

Figure 2 : exemple d'acte : réduction de fracture du fémur (extrait de https://www.ameli.fr/accueil-de-la-ccam)

Trois types d'informations seront clairement distingués dans cet article:

- Les diagnostics principaux (DP) sont supposés être assez facilement codés (par la CIM-10) par les professionnels de la santé.

- Les diagnostics secondaires (DS) sont parfois difficiles à recueillir mais il est important de les noter pour obtenir des informations exhaustives sur les soins prodigués. Ils sont également codés dans la CIM-10.

- D'autres caractéristiques telles que des informations administratives ou cliniques sont contenues dans la base de données PMSI, y compris les actes médicaux encodés dans la CCAM.

Deux échelles de bases de données ont été utilisées lors des travaux de recherche : nous utilisons d'abord certaines données à l'échelle locale des hôpitaux, afin de former et d'évaluer les méthodes d'apprentissage automatique. Une base de données régionale a également été utilisée pour comparer l'efficacité des méthodes proposées à plus grande échelle. Les résultats détaillés dans ce document proviennent de la base de données PMSI locale, nous disposions pour la période d'étude d'environ 90000 épisodes d'hospitalisation. Ce travail a été réalisé en étroite collaboration avec le DIM, pour accéder et comprendre la base de données PMSI ainsi que pour élaborer les choix nécessitant une expertise médicale. 


\section{Etat de l'art}

\subsection{Codage automatique de diagnostics médicaux}

L'utilisation de terminologies standards est très importante pour attribuer des codes non ambigus à certaines informations médicales complexes afin d'améliorer le traitement informatique des données médicales en vue de d'améliorer l'interprétabilité, la synthèse, les rapports, etc. Les professionnels de santé effectuent ces tâches en étudiant attentivement le contenu des dossiers des patients et l'informatisation de ce type de démarche est proposée par certaines approches ayant pour objectif de coder automatiquement le/les diagnostics à partir de données médicales. La plupart des travaux proposés dans la littérature utilisent des systèmes d'apprentissage automatique sur une grande quantité de données des dossiers patient, comprenant du texte, très présent dans les informations médicales. Des travaux portant sur l'annotation des textes en utilisant des méthodes de text mining sont actuellement explorés, donnant lieu à de larges campagnes d'évaluation telles $\mathrm{CLEF}^{1}$ (Roberts et al, 2007) ou semEVAL ${ }^{2}$. L'encodage automatique des diagnostics à l'aide des données contenues dans les différents dossiers patient a été proposé dans la littérature et plusieurs travaux proposant de telles approches sont réalisés à partir des données MIMIC ${ }^{3}$, une base de données médicales open source. Les recherches de (Perotte et al., 2014) menées sur cette base fournissent un travail de référence à la communauté dans le cadre de la prédiction du code de diagnostic. Différents types de classifications ont été proposés (par exemple, Kavuluru et al., 2015, Baumel et al., 2017, Karmakar, 2018), y compris les méthodes de réseau neuronal. En terme de sélection des variables, Scheurwegs et al. (2017) proposent une méthode de sélection basée sur la couverture (coverage-based) afin d'améliorer la sélection des caractéristiques.

Comme nous n'utilisons pas d'autres informations que les données PMSI, nous ne disposons pas de texte médical contrairement aux travaux que nous venons d'exposer. L'originalité de cette approche fait que l'utilisation directe des méthodologies proposées pour le codage diagnostique n'est pas pertinente et nous avons exploré les méthodes de sélection de caractéristiques en général.

\subsection{Feature selection (FS)}

La sélection de variables est une technique d'apprentissage automatique qui consiste à sélectionner des attributs pertinents et à ignorer les attributs non pertinents ou redondants pour décrire clairement un problème avec une dégradation minimale des performances (Guyon, 2006, Bolón-Canedo et al. 2015). Ce processus

\footnotetext{
${ }^{1}$ http://clef2019.clef-initiative.eu/

${ }^{2}$ http://alt.qcri.org/semeval2019/

${ }^{3}$ https://mimic.physionet.org/
} 
d'apprentissage machine est de plus en plus demandé dans le contexte du big data où plusieurs questions doivent être abordées (Liu et al. 2017) comme l'évolutivité, le traitement en temps réel, le nombre explosif de dimensions, le coût des fonctions et la sélection des fonctionnalités distribuées.

Utiliser une méthode de FS conduit à réduire considérablement la dimensionnalité des problèmes. Soulignons que cette technique est complètement différente des techniques d'extraction d'attributs, qui consistent à trouver l'ensemble le plus compact et informatif d'attributs (Bolón-Canedo et al. 2015), comme les méthodes LDA - Linear Discriminant Analysis ou PCA - Principal Component Analysis (Martinez and Kak, 2001). Les méthodes de sélection des attributs peuvent être classées dans les catégories de filtrage ou de méthodes intégrées (embedded, wrappers).

Les méthodes de filtrage s'appliquent indépendamment des méthodes de machine learning (classifieur) pour la sélection de features en se basant sur des analyses descriptives statistiques. Les méthodes intégrées, quant à elles, utilisent des classifieurs pour l'aide à la sélection de l'ensemble de sous-features pertinentes. Ces méthodes de sélection peuvent être également vues sous l'angle univarié ou multivarié en fonction du nombre de features mis en jeu. Les méthodes univariées telles que Chi-squared (Setiono and Liu, 1995) ou Fisher-score (Duda et al. 2000) étudient les caractéristiques statistiques d'une seule feature. Quant aux méthodes multivariées, plusieurs features sont étudiées en même temps telles les techniques RefliefF (Kononenko, 1994), CFS (Hall, 1999) ou INTERACT (Zhao and Liu, 2007).

Le domaine médical est caractérisé par une grande variété de ressources de données telles que les -omiques, les images médicales, les dossiers patients, les données médico-économiques, les rapports textuels, etc. En ce qui concerne, les jeux de données construits à partir des données médicales, il existe trois principales catégories de caractéristiques décrivant ces jeux de données:

- Jeux de données composés de très petits échantillons et d'un très grand nombre de fonctionnalités, tels que DNA Microarray. Par exemple, les ressources de Microarray sont composées de très petits échantillons, souvent moins de 100 patients (Bolón-Canedo et al. 2015) et d'un très grand nombre de caractéristiques dues à l'expression des gènes. Un tel dataset peut avoir de 6000 à 60000 caractéristiques le décrivant.

- Jeux de données composés de très grands échantillons et d'une taille moyenne de fonctionnalités. Des exemples de tels jeux de données sont les ressources textuelles (rapports hospitaliers) et les ensembles de données d'Imagerie médicale. Ces corpus, sont structurellement comparables à ceux proposés par de nombreuses campagnes d'évaluation comme CLEF ou SemEVAL, sont souvent de taille réduite par rapport à ces campagnes.

- Jeux de données composés à la fois d'échantillons de taille moyenne et de fonctionnalités de taille moyenne. Le ratio échantillons / caractéristiques approche 
0,04 ( 34000 caractéristiques / 700000 exemples). L'ensemble des données PMSI s'inscrit dans ce cas typique.

Ces caractéristiques sont étroitement liées aux défis bien connus des techniques de FS, à savoir problèmes de petite taille, données déséquilibrées, caractéristiques d'instabilité et sélection de caractéristiques dépendant de la classe (Wang et al. 2016). Dans la section suivante, nous allons nous focaliser sur le problème de stabilité dans les méthodes de FS.

\subsection{Méthodologies de sélection de variables stables}

\subsubsection{Stabilité des variables}

Deux types de caractéristiques de stabilité peuvent être distingués:

-Stabilité des variables par rapport à la méthode de sélection des variables. Ce premier type de stabilité est en jeu lors de l'obtention de différentes variables avec différentes méthodes FS appliquées sur le même jeu de données. Ce type d'instabilité est étudié dans le contexte de la découverte de biomarqueurs à partir de données génomiques (Jovic et al. 2015, Haury et al., 2011, He et Yu, 2010). Le document conclut que les résultats des méthodes univariées semblent être plus stables que les méthodes multivariées pour les données génomiques.

-Stabilité des variables dépendant des jeux de données d'apprentissage. Ce deuxième type d'instabilité se produit lorsque différentes variables sont sélectionnées sur des jeux de données d'apprentissage légèrement différents avec une même méthode FS utilisée. «Stability of a feature selection algorithm can be viewed as the consistency of an algorithm to produce a consistent feature subset when new training samples are added or when some training samples are removed» (Chandrashekar et Sahin, 2014). Ce problème est souligné par (Dunne et al., 2002, Yang et Mao, 2011) qui montrent des exemples d'instabilités en exécutant plusieurs fois l'algorithme de sélection de variables et en modifiant le jeu d'apprentissage en ajoutant ou en supprimant des exemples. Si l'algorithme produit un sous-ensemble différent pour chaque exécution, l'algorithme peut ne pas être fiable pour la sélection des variables.

\subsubsection{Méthodologies}

De nombreuses méthodologies sont disponibles pour générer des sélections de variables stables. Les grandes familles de méthodes de FS décrites dans la section 3.2 sont utilisées dans ces méthodologies :

- Sélection de variables par ensembles : ces méthodologies combinent plusieurs modèles appris tels que des approches de bagging ou de boosting. Les principales étapes consistent alors à : (i) créer un ensemble de sélecteurs de variables différentes et (ii) agréger les résultats en résolvant un problème d'agrégation basé sur les rangs (Seijo-Pardo et al. 2017). Dans de telles méthodologies de sélection, il existe deux 
stratégies pour construire divers apprenants locaux : (i) perturbation des données en utilisant différents échantillonnages générés à partir du jeu de données d'origine avec une même méthode de sélection et (ii) de façon opposée, la fonction de perturbation utilise différents algorithmes de FS sur un même jeu de données.

- Sélection des variables avec pertinence des variables antérieures : ces méthodologies supposent que toutes les caractéristiques sont également pertinentes et que certaines connaissances préalables seront acquises soit par des experts du domaine, soit par des méthodes par transfert pour l'apprentissage.

- Sélection de variables de groupe : l'idée principale de cette approche est de détecter un groupe de caractéristiques robustes insensibles aux variations des échantillons d'apprentissage. Ces méthodologies s'articulent en deux étapes : (i) la génération de variables de groupe, pilotée par des experts ou pilotée par des données en utilisant des techniques de clustering ; (ii) la transformation de groupes de variables est un processus d'homogénéisation de celles-ci dans une variable plus compacte (par exemple en utilisant PCA).

\subsubsection{Evaluation de la stabilité}

La stabilité des méthodes de FS peut être mesurée en quantifiant la sensibilité de la sélection aux variations du jeu d'apprentissage (He et $\mathrm{Yu}, 2010$ ). Plus concrètement, la stabilité sera évaluée en comparant les sous-ensembles de variables qui en résultent (par exemple comparer le rapport de l'intersection, de l'union), des classements (ranking) ou en calculant un score de pondération.

Certaines propositions visant à minorer l'instabilité des variables ont été identifiées dans la littérature. Dunne et al. (2002) ont étudié l'instabilité des méthodes wrapper et proposé d'utiliser la «distance de Hamming» pour mesurer l'instabilité des variables. Ils ont proposé d'atténuer l'instabilité en répétant la méthode wrapper plusieurs fois et en conservant les éléments les plus fréquents. Somol et Novovi (2010) ont étudié diverses mesures de stabilité; des variables plus robustes peuvent être sélectionnées à partir de différents jeux de données en utilisant ces mesures.

En considérant les travaux de la littérature, notre proposition est dérivée des méthodes de FS de type ensemble. Quelle que soit la méthode FS, nous pouvons obtenir un ensemble combiné de variables en partant de différents sous-jeux de données échantillonnés obtenus à partir du jeu de données d'origine. En ce qui concerne la stabilité, notre méthode est axée sur la stabilité de la FS pour une perturbation des jeux de données, en utilisant différents échantillons d'apprentissage et en convergeant vers un nombre stable de jeux de données échantillonnés.

Pour les méthodologies d'ensemble, nous citons le travail de (Yang et Mao, 2011, Yang et al., 2004). Des approches de moyenne bayésienne sont proposées dans (Yeung et al., 2005). Le renforcement a été adapté pour améliorer la robustesse et la stabilité des caractéristiques finales (Ben-Dor et al., 2000, Dudoit et al., 2002). De façon générale, les méthodes d'ensemble produisent des variables robustes et 
stables, le principal inconvénient de ces méthodes étant les besoins en ressources de calcul supplémentaires qui, dans certains cas, ne sont pas envisageables lorsque d'énormes jeux de données sont utilisés.

\section{Une approche pour sélectionner des features caractéristiques stables à partir des informations médico-économiques issues du PMSI}

L'approche proposée s'inscrit dans un objectif global qui consiste à prédire un diagnostic secondaire (DS), connaissant le diagnostic principal (DP) et d'autres caractéristiques codées dans le PMSI (âge, mode d'entrée, actes médicaux effectués, etc.). Cette section est organisée en trois sous-sections. Dans la première, nous décrivons les matériaux utilisés à savoir les jeux de données et les méthodes. La deuxième sous-section sera consacrée à la présentation de l'approche. Dans la troisième sous-section, nous présentons les résultats de l'évaluation de l'approche.

\subsection{Matériel et méthodes proposés}

Comme nous l'avons déjà mentionné, prédire le diagnostic en utilisant uniquement la base de données PMSI est assez original et présente de fortes contraintes en raison du nombre limité d'informations contextuelles dans une telle base. Nous avons décidé de construire les jeux de données sur la connaissance du diagnostic principal, en effet :

- le DP est plus facile à trouver par le codeur que le/les DS, il peut être considéré comme une information fiable.

- Prédire les diagnostics a été montré comme très difficile si l'on considère une base médicale en général, même lorsqu'il y a utilisation d'informations riches telles que les informations, y compris textuelles, des dossiers médicaux. De meilleurs résultats ont été obtenus en se concentrant sur un domaine médical spécifique. Dans notre cas, construire des sous-ensembles liés à un DP apparaît comme une hypothèse raisonnable compatible avec les contraintes PMSI et permettant une focalisation sur le domaine médical de ce diagnostic.

- La connaissance du DP peut guider la transformation de variables continues en informations qualitatives (par exemple pour un DP, l'âge moyen ou le nombre moyen d'actes sont connus). Dans notre cas, cette connaissance permet de discrétiser ce type d'information en «moins que la moyenne $-2 *$ sd (écart-type) », " entre la moyenne- $2 *$ sd et la moyenne $+2 * s d », \ll$ plus que la moyenne $+2 * s d »$.

Les données PMSI ont été prétraitées pour disposer des informations permettant de mettre en œuvre les méthodes de ML. Les données continues ont été discrétisées en fonction du DP retenu, comme mentionné précédemment. Le codage médical (actes et diagnostics) a été mis à un niveau de granularité qui a été choisi selon une étude mentionnée dans (Chahbandarian et al. 2016). Au total, nous avons utilisé 183 variables pour construire notre modèle de ML. 
Prédire un diagnostic secondaire en partant de l'hypothèse que nous connaissons le diagnostic principal (le DP) est une hypothèse, assez forte, et cependant elle est confortée par l'analyse de l'activité de codage (interview et observations) que nous avons menée (Chahbandarian, 2017, chap5). Les DP étaient rapidement trouvés et pour les DS, une boucle de travail se mettait en place, comportant l'élaboration de DS suite à un indice repéré dans les informations médicales (exemple un matelas anti-escarre est prescrit $\Rightarrow$ y a-t-il escarre ?), suivi de recherches approfondies dans les dossiers pour infirmer ou confirmer cette hypothèse de DS (escarre avéré ou non suivant les autres documents).

\subsubsection{Description des jeux de données}

Comme nous l'avons déjà présenté, le nombre de codes de diagnostic est très important: nous avons environ 34000 codes complets en CIM-10 (version française ICD-10) et environ 2000 codes si nous nous référons aux 3 caractères codant de manière plus générale les différentes maladies. Si nous prenons en compte tous les documents contenus dans la base de données PMSI de l'hôpital, sachant que cet hôpital local traite diverses maladies, nous devons faire face à la rareté de chaque code dans ces bases de données. A fortiori un couple (DP-DS) peut très souvent être rare. Pour réduire la rareté des couples (DP-DS) dans l'ensemble de la base de données PMSI, nous avons donc choisi de travailler par sous-ensemble de données répondant à notre hypothèse de DP connu. Nous avons ainsi pu nous focaliser sur quelques sous-ensembles, sélectionnés à priori, cela ne remet pas en cause le principe de la démarche qui pourrait se transposer à tous les sous-ensembles mais cela nous permet d'aller au bout d'une démarche de compréhension et d'interprétation des résultats obtenus.

Par conséquent, en collaboration avec le médecin responsable du département DIM, nous avons choisi des ensembles de données particulièrement intéressants à étudier. Pour gérer ce choix,

- nous avons identifié quelques DS intéressants à détecter et à étudier pour le DIM : ce sont des DS qui sont assez impactants pour la prise en charge du traitement global.

- Une fois ce choix effectué, nous avons trouvé dans la base PMSI les DP les plus fréquents qui sont associés à ces DS tout en présentant un intérêt médical à être examinés (ces couples DP-DS ne sont pas trop évidents : ils n'appartiennent pas à des cas très bien connus des professionnels de santé). L'utilisation de méthode de ML pour prédire les DS sachant ces DP présente alors un intérêt pour les professionnels de santé.

- Puis pour chacun de ces DP, nous avons extrait de la base PMSI complète étudiée, la sous-base de données composée de tous les éléments contenant ce DP (nous appellerons ce jeu de données le DatasetDP).

En suivant cette méthode, 14 DatasetDP intéressants ont été construits. Chacun de ces datasetDP est composé de cas positifs (DP + le DS visé) et négatifs (DP sans le DS ciblé). Ces 14 datasetDP sont présentés dans le Tableau 1. Les colonnes DS et 
DP présentent le code et la description du couple DP-DS retenu ; la colonne count DP informe sur le nombre d'enregistrements dans le dataset qui sont tous les couples (DP, *) ; la colonne pos.Count donne le nombre de couples d'enregistrements positifs (DP,DS) et la colonne imbalance ratio indique le rapport de déséquilibre entre cas positifs et cas négatifs soit le nombre de (DP,DS) sur le nombre de couples (DP, autres que DS). Sur le tableau 1, on observe le rapport déséquilibré particulièrement faible des couples choisis (DP,DS) : $71 \%$ des ensembles de données ont un ratio de déséquilibre inférieur ou égal à 10\%, les autres sont proches de $30 \%$.

TAB. 1 Les 14 DatasetDP retenus pour étudier les couples (DP,DS).

\begin{tabular}{|c|c|c|c|c|}
\hline DS & DP & $\begin{array}{l}\text { DP } \\
\text { Count }\end{array}$ & $\begin{array}{l}\text { Pos. } \\
\text { Count }\end{array}$ & $\begin{array}{l}\text { Imbal } \\
\text { ance } \\
\text { ratio }\end{array}$ \\
\hline R26: Abnormalities of gait and mobility & F05: Delirium & 540 & 73 & $14 \%$ \\
\hline E44: Protein-calorie malnutrition & F05: Delirium & 540 & 137 & $25 \%$ \\
\hline E66: Overweight and obesity & I48: Atrial fibrillation and flutter & 961 & 91 & $9 \%$ \\
\hline R26: Abnormalities of gait and mobility & I50: Heart failure & 2428 & 104 & $4 \%$ \\
\hline R26: Abnormalities of gait and mobility & J15: Bacterial pneumonia & 694 & 70 & $10 \%$ \\
\hline E44: Protein-calorie malnutrition & J18: Pneumonia & 1201 & 72 & $6 \%$ \\
\hline R26: Abnormalities of gait and mobility & J20: Acute bronchitis & 879 & 88 & $10 \%$ \\
\hline R26: Abnormalities of gait and mobility & J96: Pneumonitis & 272 & 80 & $29 \%$ \\
\hline E66: Overweight and obesity & K80: Cholelithiasis & 1114 & 75 & $7 \%$ \\
\hline E66: Overweight and obesity & R10: Abdominal and pelvic pain & 2650 & 95 & $4 \%$ \\
\hline E44: Protein-calorie malnutrition & $\begin{array}{l}\text { R29: Symptoms and signs } \\
\text { involving the nervous and } \\
\text { musculoskeletal systems }\end{array}$ & 446 & 76 & $17 \%$ \\
\hline E44: Protein-calorie malnutrition & $\begin{array}{l}\text { R41: Symptoms and signs } \\
\text { involving cognitive functions and } \\
\text { awareness }\end{array}$ & 669 & 67 & $10 \%$ \\
\hline R26: Abnormalities of gait and mobility & R50: Fever & 1184 & 68 & $6 \%$ \\
\hline L89: Pressure Ulcer & S72: Fracture of femur & 1110 & 25 & $2 \%$ \\
\hline
\end{tabular}

\subsubsection{Les méthodes utilisées}

Deux solutions sont possibles pour traiter le problème des données déséquilibrées ou des données asymétriques : soit le sur-échantillonnage, soit le sous-échantillonnage. Nous avons choisi la méthode de sous-échantillonnage principalement en raison des données médicales que nous traitons. La méthode de sur-échantillonnage a l'inconvénient de simuler des cas de figures non réelles ou improbables pour les patients. La méthode de sous-échantillonnage mise en place a l'avantage de nécessiter beaucoup moins de puissance de calcul. Une étude préliminaire (Chahbandarian, 2017) montre que les meilleurs résultats ont été obtenus avec cette méthode lors de l'utilisation de notre jeu de données.

Concernant la méthode de FS, nous avons testé la qualité rendue par deux méthodes de type Filter (univariée et multivariée) sur nos jeux de données. Les méthodes de type Filter passent à l'échelle et sont très rapides ce qui est conforme à une meilleure optimisation des ressources. Pour notre étude, nous avons retenu deux méthodes de caractéristiques différentes, ceci nous permet de comparer les résultats 
obtenus dans ces deux cas alors même que nous avions vérifié que toutes deux produisent des modèles d'apprentissage de bonne qualité pour notre étude de cas :

- GainR (Witten et al., 2005) permet l'évaluation et la sélection des variables individuellement sans tenir compte de l'influence et de la corrélation par rapport aux autres variables. C'est une méthode univariée. Néanmoins, cette méthode réduit le biais en faveur de la sélection de variables à plusieurs valeurs. Les attributs sont classés en fonction du score GainR. Les entités ayant un score supérieur à un seuil défini sont conservées.

- CFS (Hall, 1999) évalue les variables en tant qu'ensemble. C'est une méthode multivariée. La méthode CFS classe les sous-ensembles d'entités en fonction du degré de redondance parmi les entités. Elle recherche des sous-ensembles de variables qui sont individuellement bien corrélées avec la classe, mais qui ont une faible corrélation entre elles. CFS est souvent combinée avec des stratégies de recherche comme : forward selection, backward elimination, recherche bidirectionnelle et recherche génétique. La formule de calcul de corrélation utilisée pour CFS est la suivante:

$$
r_{z c}=\frac{k \overline{r_{z i}}}{\sqrt{k+k(k-1) \overline{r_{i i}}}}
$$

où :

- $\quad r_{z c}$ est la corrélation entre le sous-ensemble de features et la classe cible,

- $\quad k$ est le nombre de sous-ensemble de features,

- $\quad r_{z i}$ est la moyenne des corrélations entre les sous-ensembles de features et la classe cible,

- $\quad r i$ est la moyenne d'inter-corrélation entre les sous-ensembles de features.

Selon (Bolón-Canedo et al., 2015), GainR et CFS sont recommandées pour les problèmes ayant un nombre de variables dépassant le nombre d'échantillons. (BolónCanedo et al., 2015) suggère que «no-size-fits all ». Nous observons surtout que les méthodes multivariées ont tendance à générer de meilleurs résultats que les méthodes univariées, à quelques exceptions près pour certains jeux de données génériques (Jovic et al., 2015, Haury et al., 2011, He et Yu, 2010). Néanmoins, le coût de calcul des méthodes multivariées est encore plus important que celui des méthodes univariées.

\subsection{L'approche proposée}

$\mathrm{Si}$ nous rappelons les deux questions principales traitées dans cet article: «Quelle est la stabilité des variables lorsqu'une méthode FS est appliquée sur un jeu de données échantillonné? » et «Combien de jeux de données sont nécessaires pour sélectionner des variables stables », en supposant que les jeux de données sont pré-traités et prêts à être explorés, nous devons clarifier si les variables 
sélectionnées sont différentes chaque fois que le jeu de données est échantillonné ; et regarder si un nombre de variables stables assez significatif est trouvé pour ces variables stables communes aux jeux de données échantillonnés. Si de telles caractéristiques stables sont trouvées, la question qui reste à valider est "Ces variables stables sont-elles de bonne qualité?".

\subsubsection{Trouver les variables fiables}

Notre approche, présentée Figure 3, s'inscrit parmi les méthodes d'ensemble car nous nous appuyons sur la convergence des méthodes de FS sur plusieurs jeux de données échantillonnées jusqu'à la stabilisation d'un ensemble de variables pertinentes.

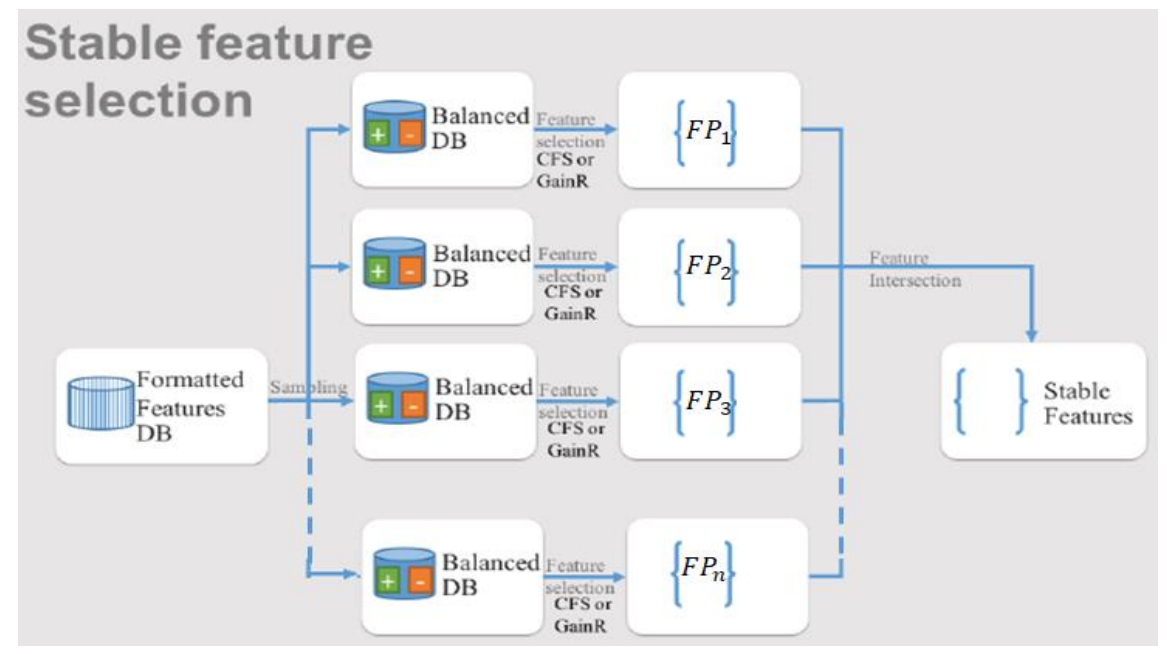

Figure 3. Sélection de variables stables au travers du processus itératif.

Pour chacun des 14 DatasetDP identifiés pour l'étude, les variables stables sont obtenues de la façon suivante :

- Initialisation des Features Stables à l'ensemble de toutes les Features pour le DatasetDP considéré, puisque les features ne sont pas encore sélectionnées $($ FStables $=\{$ ensemble de $F\})$

- Réduction de l'ensemble des Features Stables de façon itérative (Figure3), le processus est réitéré avec à chaque fois un nouvel échantillonnage, jusqu'à ce que l'approche converge.

- Récupération d'un ensemble de features pertinentes à l'étape i $(F P i)$. Ceci est fait à travers l'échantillonnage de la base de données et l'utilisation d'une méthode de FS: l'échantillonnage est utilisé pour équilibrer les exemples positifs et négatifs ; les caractéristiques pertinentes sont détectées 
à l'aide de la méthode de FS retenue (les deux méthodes CFS et GainR seront explorées).

- Mise à jour des Features Stables. Ceci est fait via l'intersection des Features Stables et des features pertinentes trouvées pour l'échantillon. FStables $=$ FStables $\cap F P i$

Si l'ensemble des Features Stables alors obtenu n'est pas vide, un ensemble de variables communes stable et pertinent est détecté. L'évolution de l'ensemble commun de variables est ensuite soigneusement étudiée à travers le nombre de variables qu'il contient. Cela permet d'évaluer si proposer une telle intersection entre les différentes étapes permet de trouver une convergence pour les variables sélectionnées. Si c'est le cas, l'objectif est de déterminer combien de variables peuvent être détectées comme étant stables et combien d'itérations sont nécessaires pour détecter l'ensemble stable des variables.

\subsubsection{Evaluation de la qualité}

Nous allons chercher à évaluer la pertinence des variables retenues à l'aide d'une évaluation de la prédiction des diagnostics secondaires connaissant le DP. Nous construisons un modèle de ML construit sur la base d'un jeu de données retenant l'ensemble stable de variables. Nous examinons alors la qualité de la prévision proposée qui va informer sur la qualité et le pouvoir prédictif des variables sélectionnées.

\subsection{Résultats}

Dans cette section, nous présentons les résultats obtenus à partir de la base de données PMSI.

Nous avons mis en œuvre l'approche décrite ci-dessus à travers les choix d'implémentation indiqués dans la section 4.1: (i) la méthode de souséchantillonnage a été utilisée pour construire des jeux de données équilibrés en termes d'exemples positifs et négatifs; Pour GainR, le seuil choisi a été défini empiriquement afin de conserver peu de variables. Avec un seuil de 0,02, GainR conserve en moyenne 20 variables.

\subsubsection{Résultats pour la stabilité des variables sélectionnées}

Lors de cette première évaluation, nous analysons la stabilité des entités sélectionnées par rapport au nombre de jeux de données échantillonnés utilisés. Pour ce faire, nous exécutions $14 \times 5$ fois (14=le nombre de couples DP-DS) le processus en augmentant le nombre de jeux de données échantillonnés de 1 à 5 . Pour chaque itération, nous comptons les variables communes générées par la méthode FS pour chacun des jeux de données. 
Les résultats pour les 14 diagnostics retenus dans l'étude sont présentés dans la figure 3 : la figure $3 a$ présente le cas où la méthode GainR est utilisée et la figure $3 b$ le cas où la méthode CFS est utilisée.

Les résultats indiquent que le nombre d'entités communes diminue significativement lorsque plusieurs jeux de données sont utilisés, ce qui indique que les entités détectées via un seul jeu de données d'échantillonnage ne sont pas suffisamment stables et que plusieurs jeux de données doivent être étudiés. De plus, les résultats montrent que le nombre de variables communes devient stable lorsque différents jeux de données sont utilisés, ce qui signifie que les variables communes des jeux de données échantillonnés indépendamment contiennent des variables stables qui apparaissent dans tous les jeux de données échantillonnés.

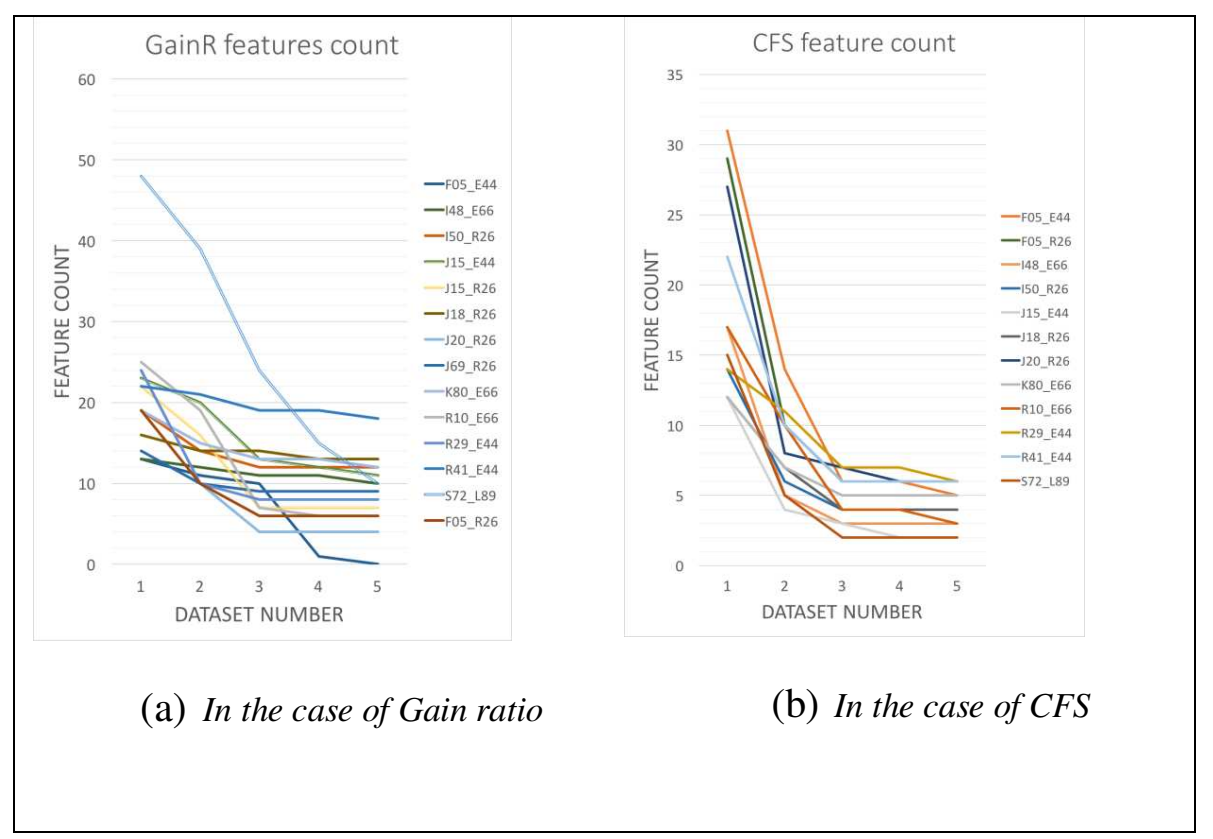

Figure 4. La relation entre le nombre de dataset (axe $x$ ) et le nombre de features issues communs identifiés (axe y) pour ces datasets.

Cependant, dans un cas (dans la figure 4a, 5 jeux de données pour le couple F05E44 : Delirium, non induit par l'alcool et d'autres substances psycho-actives Malnutrition protéino-énergétique légère ou modérée) il n'y a pas de variable commune ce qui signifie que, pour GainR, la nature du (DP-DS) induit une sélection de caractéristiques très variable selon les échantillons.

À partir des résultats, nous pouvons également observer que la méthode multivariée CFS est meilleure que la méthode univariée GainR pour notre jeu de données. La première présente des courbes similaires, indépendamment du couple 
DP-DS retenu, la seconde semble être impactée par les instances du jeu de données. Nous retrouvons ainsi des caractéristiques remarquées lors de la comparaison de ces méthodes dans la littérature.

En suivant notre approche, le nombre de jeux de données requis pour sélectionner un ensemble stable d'entités peut être déterminé comme le nombre d'échantillonnages nécessaire pour obtenir une convergence du nombre d'entités communes sélectionnées. En comparant les deux figures 4 (a) et 4 (b) dans le cas de la base de données PMSI, trois est le nombre minimal de jeux de données à échantillonner pour obtenir des variables stables.

\subsubsection{Résultats sur la qualité des variables sélectionnées}

Nous supposons que les variables communes sélectionnées pour trois jeux de données échantillonnés contiennent les variables les plus pertinentes pour la prédiction, et excluent les entités qui sont bruitées et moins pertinentes pour la prédiction.

Pour étayer notre affirmation, nous évaluons les résultats de qualité des variables sélectionnées, en évaluant la précision des modèles de prédiction construits à partir de ces informations.

Nous avons utilisé l'arbre de décision CART pour construire un modèle de prédiction. Cette méthode appartient aux class-labeled training tuples. Nous avons choisi une méthode d'arbre de décision car elle génère des modèles simples, elle est facile à interpréter et elle peut ainsi être validée par des médecins qui ne sont pas nécessairement des experts en informatique. En outre, cette méthode est évolutive et peut produire des modèles efficaces même lorsque de grandes quantités de données sont utilisées (Cieslak \& Chawla 2008). Par conséquent, nous avons utilisé CART pour construire un modèle qui code les diagnostics secondaires en utilisant les 14 ensembles de données préparés présentés dans le tableau 1 et en retenant les variables stables sélectionnées en utilisant notre approche.

Nous avons utilisé F1, Recall, Precision et l'Area Under ROC Curve (AUC) pour mesurer la performance du modèle d'encodage. Les résultats de l'évaluation sont présentés dans le tableau 2.

Les évaluations présentées dans ce tableau indiquent que les deux méthodes (CFS-GainR) produisent des modèles de prédiction avec des mesures de performance ayant des moyennes similaires, la méthode CFS est légèrement meilleure, même si elle présente moins de variables stables. Cela semble indiquer que la méthode GainR produit peu de variables redondantes sans affecter sa performance. Les deux méthodes FS produisent un bon modèle de prédiction mais avec un nombre de variables différent. Le choix de la méthode de FS sera alors une simple question de préférence selon les cas d'utilisation retenus. 
TAB. 2 Evaluation de la qualité de stabilité des features en terme de performance des résultats de prédiction de l'algorithme CART Decision Tree.

\begin{tabular}{|c|c|l|l|l|l|l|l|l|}
\hline & \multicolumn{1}{|c|}{} & \multicolumn{3}{|l|}{} \\
\hline DP-DS & F1 & AUC & Prec & Rec & F1 & AUC & Prec & Rec \\
\hline F05_R26 & $58 \%$ & $66 \%$ & $70 \%$ & $50 \%$ & $64 \%$ & $65 \%$ & $65 \%$ & $63 \%$ \\
\hline I48_E66 & $59 \%$ & $61 \%$ & $63 \%$ & $56 \%$ & $55 \%$ & $64 \%$ & $73 \%$ & $44 \%$ \\
\hline I50_R26 & $61 \%$ & $59 \%$ & $69 \%$ & $55 \%$ & $70 \%$ & $73 \%$ & $77 \%$ & $65 \%$ \\
\hline J15_E44 & $83 \%$ & $86 \%$ & $100 \%$ & $71 \%$ & $78 \%$ & $71 \%$ & $64 \%$ & $100 \%$ \\
\hline J15_R26 & $71 \%$ & $75 \%$ & $71 \%$ & $71 \%$ & $81 \%$ & $72 \%$ & $72 \%$ & $93 \%$ \\
\hline J18_E44 & $56 \%$ & $64 \%$ & $64 \%$ & $50 \%$ & $61 \%$ & $50 \%$ & $50 \%$ & $79 \%$ \\
\hline J20_R26 & $64 \%$ & $74 \%$ & $100 \%$ & $47 \%$ & $67 \%$ & $69 \%$ & $69 \%$ & $65 \%$ \\
\hline J69_R26 & $58 \%$ & $70 \%$ & $88 \%$ & $44 \%$ & $67 \%$ & $66 \%$ & $54 \%$ & $88 \%$ \\
\hline K80_E66 & $71 \%$ & $60 \%$ & $56 \%$ & $100 \%$ & $71 \%$ & $60 \%$ & $56 \%$ & $100 \%$ \\
\hline R10_E66 & $67 \%$ & $66 \%$ & $65 \%$ & $68 \%$ & $62 \%$ & $62 \%$ & $54 \%$ & $74 \%$ \\
\hline R29_E44 & $79 \%$ & $76 \%$ & $72 \%$ & $87 \%$ & $69 \%$ & $69 \%$ & $65 \%$ & $73 \%$ \\
\hline R41_E44 & $54 \%$ & $54 \%$ & $54 \%$ & $54 \%$ & $73 \%$ & $77 \%$ & $89 \%$ & $62 \%$ \\
\hline R50_R26 & $67 \%$ & $70 \%$ & $64 \%$ & $69 \%$ & $69 \%$ & $68 \%$ & $58 \%$ & $85 \%$ \\
\hline S72_L89 & $40 \%$ & $40 \%$ & $40 \%$ & $40 \%$ & $75 \%$ & $80 \%$ & $100 \%$ & $60 \%$ \\
\hline
\end{tabular}

\section{Discussion}

L'utilisation de la base de données PMSI pour prédire les diagnostics est intéressante car le PMSI est une base médicale standardisée, nationale, exhaustive et fiable. Cependant, réaliser des travaux de ML sur une telle base reste très difficile car cette dernière n'intègre pas beaucoup de données médicales contextualisées. A titre indicatif, le PMSI ne contient aucune ordonnance, aucune lettre médicale et garde les informations anonymes. Il était ainsi intéressant d'étudier la capacité d'utiliser l'apprentissage automatique pour prédire les diagnostics secondaires dans un tel cadre qu'on qualifie de «minimaliste». Le problème de la sélection des variables à considérer pour mettre en œuvre les modèles de ML est l'un des principaux problèmes à résoudre. Notre étude a montré que nous pouvons proposer un ensemble de variables stable obtenues assez facilement car seulement 3 itérations sont nécessaires. Cette sélection de variables contient suffisamment d'informations pour permettre une bonne qualité de prédiction pour les diagnostics.

La force de notre approche est de fournir une approche générique indépendante des méthodes d'échantillonnage, des méthodes de FS ou des algorithmes d'apprentissage. Plusieurs méthodes d'échantillonnage peuvent être utilisées pour équilibrer l'ensemble de données, toute méthode FS peut être utilisée pour sélectionner des variables, et n'importe quelle méthode de classification pourrait être utilisée pour construire un modèle de prédiction. 
De plus, nous avons fait une étude (Chahbandarian, 2017), non développée dans cet article, qui montre que le rapport d'équilibre entre les exemples négatifs et positifs dans les différents ensembles de données n'influence pas la qualité des variables stables.

Un autre point fort est que le nombre d'entités stables est relativement faible et qu'il est donc relativement facile d'informer les professionnels de santé des règles de décision issues du modèle de décision, afin qu'ils puissent comprendre et suivre attentivement les données en jeu dans de telles règles. Nous arrivons ainsi à rendre explicite des connaissances sur les variables du PMSI qui doivent être soigneusement examinées pour un DP donné.

La faiblesse de l'approche est qu'elle ne considère pas le niveau sémantique de ces variables médicales, alors que cela pourrait améliorer la compréhension des relations entre les variables. De ce fait, en matière de qualité de prédiction, l'approche proposée ne concurrence pas les méthodes qui utilisent des données non structurées (généralement du texte) en entrée. Même si celles-ci ne connaissent aucun code de diagnostic lors de la prédiction, elles disposent d'une plus grande richesse d'informations, et il a été établi que fournir des données structurées mais également non structurées peut conduire à une meilleure prédiction (Scheurwegs $e t$ al., 2016).

En évaluant les modèles obtenus au niveau local sur des données au niveau régional, nous avons validé la généricité de notre approche. La diversité de l'information médicale qui existe entre les hôpitaux fait que les résultats obtenus localement peuvent être moins efficaces à un niveau plus global. En effet, tous les hôpitaux n'ont pas les mêmes populations de patients et les résultats obtenus peuvent différer d'un hôpital à un autre.

Notre approche constitue une alternative utilisant des sources structurées contenant de nombreuses informations et facilement accessibles, la précision que nous avons obtenue semble acceptable pour soutenir le travail d'encodage de diagnostics secondaires.

\section{Conclusion et perspectives}

Dans cet article, nous proposons une approche pour sélectionner des variables stables dans le contexte de la prédiction de diagnostics secondaires à partir d'un ensemble de données médico-économiques à savoir le PMSI. Nos résultats donnent de nouvelles pistes pour l'aide à l'activité d'encodage réalisée dans le département DIM des hôpitaux. L'approche proposée consiste à exploiter le paradigme ensembliste dans des jeux de données sous-échantillonnés et équilibrés afin de générer pour ces bases un ensemble stable et fiable de variables sur lesquelles peuvent s'appuyer des méthodes de prédiction. L'ensemble des variables trouvées est construit indépendamment de l'échantillon utilisé pour l'apprentissage automatique. La qualité des variables est déduite en fonction de la qualité de prédiction des algorithmes de ML. L'évaluation de notre approche sur les données de 
PMSI montre l'intérêt réel de cette proposition et ouvre le débat sur l'application du ML sur les sources de données PMSI qui sont rarement exploitées dans cette perspective par la communauté scientifique.

L'ensemble des étapes de ce projet s'est fait en discussion avec des professionnels de santé. Un travail d'évaluation au travers d'une analyse purement médicale des variables retenues pour chaque couple (DP-DS) resterait à développer pour compléter la validation via le modèle de prédiction vu en 3.2.1. Au-delà de cette évaluation, nous avons démarré un travail complémentaire nous permettant de mettre en évidence des règles de décision, basées sur les variables ainsi retenues pour prédire les diagnostics secondaires. Notre propos serait alors d'accompagner le professionnel de santé lors de son processus de codage : nous avons vu qu'il procédait en élaborant des listes de diagnostics secondaires candidats lors du parcours des dossiers, nous souhaitons y adjoindre les diagnostics prédits par notre approche tout en explicitant les variables déterminantes pour ces choix afin de garantir une explicabilité du processus de décision. Cette phase d'évaluation doit faire l'objet d'un travail ultérieur.

Pour les travaux futurs, nous continuerons à évaluer cette approche en combinant les variables sélectionnées issues de différentes méthodes de FS. Nous prévoyons aussi mener une étude comparative complète déclinant différents types de paramètres pour évaluer la stabilité. De nouvelles méthodes de FS pourraient également être testées sur les jeux de données DatasetDP.

En conservant l'idée d'un travail centré sur le PMSI, nous pourrions également explorer les résultats obtenus en utilisant des jeux de données moins spécifiques, par exemple nous pourrions essayer d'agréger des jeux de données plus larges que les jeux liés aux DP, comme par exemple retenir l'ensemble des informations liées à un service médical et explorer l'impact que cela pourrait avoir sur la qualité de prédiction diagnostique.

Dans une perspective plus large, la prédiction de diagnostics en utilisant des approches multicentriques et réintégrant le dossier patient dans les sources de données utilisées pour l'apprentissage, constitue une piste de travail intéressante. Il s'agirait alors de comparer le bénéfice obtenu en utilisant des données moins accessibles, tant parce que non anonymisées que parce que plus difficiles à traiter car plus hétérogènes et complexes.

\section{Remerciements}

Le travail de thèse de l'un des auteurs a été soutenu par un co financement region Midi-Pyrénées,IUT-Université Paul Sabatier, Technopole Castres-Mazamet, et INU Champollion. Merci aux professionnels de santé du DIM Castres-Mazamet pour leur aide précieuse. 


\section{References}

Baumel, T., J. Nassour-Kassis, M. Elhadad, and N. Elhadad (2017). Multi-label classification of patient notes a case study on ICD code assignment. CoRR abs/1709.09587.

Ben-Dor, A., L. Bruhn, N. Friedman, I. Nachman, M. Schummer, and Z. Yakhini (2000). Tissue classification with gene expression profiles. Journal of computational biology 7(3-4), 559-583.

Bolón-Canedo, V., N. Sánchez-Maroño, and A. Alonso-Betanzos (2015). Recent advances and emerging challenges of feature selection in the context of big data. Knowl.-Based Syst. 86,33-45.

Chahbandarian, G. (2017). Elicitation de l'information permanente à partir de bases de données médicales : Application au codage des diagnostics secondaires. Ph. D. thesis, ED- MITT. Financement Région Midi-Pyrénées/ IUT A-UPS / Syndicat Mixte Castres-Mazamet/ INU Champollion.

Chahbandarian, G., N. Bricon-Souf, R. Bastide, and J. Steinbach (2016). Increasing secondary diagnosis encoding quality using data mining techniques. In Tenth IEEE International Conference on Research Challenges in Information Science, RCIS 2016, Grenoble, France, 1-10.

Chandrashekar, G. and F. Sahin (2014). A survey on feature selection methods. Computers and Electrical Engineering 40(1), 16-28.

Cieslak, D. A. and N. V. Chawla (2008). Learning Decision Trees for Unbalanced Data. InMachine Learning and Knowledge Discovery in Databases, Volume 5211 LNAI, pp. 241-256. Berlin, Heidelberg: Springer Berlin Heidelberg.

Duda, R.O, Hart, P.E, and Stork, D.G. 2000. Pattern Classification (2nd Edition). Wiley-Interscience, New York, NY, USA.

Dudoit, S., J. Fridlyand, and T. P. Speed (2002). Comparison of discrimination methods for the classification of tumors using gene expression data. Journal of the American statistical association 97(457), 77-87.

Dunne, K., P. Cunningham, and F. Azuaje (2002). Solutions to instability problems with sequential wrapper-based approaches to feature selection. Journal of Machine Learning Research, 1-22.

Guyon, I. (2006). Feature Extraction: Foundations and Applications. SpringerVerlag New York, Inc.

Hall M.A, (1999). Correlation-based Feature Selection for Machine Learning, PhD Thesis, The University of Waikato, 1999.

Haury, A.-C., P. Gestraud, and J.-P. Vert (2011). The Influence of Feature Selection Methods on Accuracy, Stability and Interpretability of Molecular Signatures. PLoS ONE 6(12). 
He, Z. and W. Yu (2010). Stable feature selection for biomarker discovery. Computational Biology and Chemistry 34(4), 215-225.

Jovic, A., K. Brkic, and N. Bogunovic (2015). A review of feature selection methods with applications. 38th International Convention on Information and Communication Technology, Electronics and Microelectronics (MIPRO 2015), 1200-1205.

Karmakar, A. (2018). Classifying medical notes into standard disease codes using machine learning. CoRR abs/1802.00382.

Kavuluru, R., A. Rios, and Y. Lu (2015). An empirical evaluation of supervised learning approaches in assigning diagnosis codes to electronic medical records. Artificial Intelligence in Medicine 65(2), 155-166.

Kononenko, I. (1994). Estimating attributes: analysis and extensions of relief, in: Machine Learning: ECML-94, Springer, pp. 171-182.

Lecornu, L., G. Thillay, C. Le Guillou, P. J. Garreau, P. Saliou, H. Jantzem, J. Puentes, and J. M. Cauvin (2009). REFEROCOD: a probabilistic method to medical coding support. In Engineering in Medicine and Biology Society. EMBC 2009. Annual International Conference of the IEEE, pp. 3421-3424. IEEE.

Liu, J., Y. Lin, M. Lin, S. Wu, and J. Zhang (2017). Feature selection based on quality of information. Neurocomputing 225, 11-22.

Martinez A.M and Kak A. C (2001). PCA versus LDA," in IEEE Transactions on Pattern Analysis and Machine Intelligence, 23 (2), 228-233.

Perotte, A., R. Pivovarov, K. Natarajan, N. Weiskopf, F. Wood, and N. Elhadad (2014). Diagnosis code assignment: Models and evaluation metrics. Journal of the American Medical Informatics Association, 21(2), 231-237.

Potignon C. Musat A. Hillon P. Rat P. Osmak L. Rigaud D. Vergès B (2010). "P146-Impact financier pour les établissements hospitaliers du mauvais codage PMSI de la dénutrition et de l'au sein du pôle des pathologies digestives endocriniennes et métaboliques du CHU de Dijon" 2010.

Roberts, A., Gaizauskas, R., Hepple, M., Davis, N., Demetriou, G., Guo, Y., Kola, J., Roberts, I., Setzer, A., Tapuria, A., Wheeldin, B. (2007). The CLEF corpus: semantic annotation of clinical text. AMIA 2007 Annual Symposium proceedings. AMIA Symposium, 625-9.

Setiono R. and Liu H (1995). Chi2: Feature Selection and Discretization of Numeric Attributes, Proceedings of 7th IEEE International Conference on Tools with Artificial Intelligence (ICTAI), Herndon, Virginia, USA, pp. 388.

Scheurwegs, E., B. Cule, K. Luyckx, L. Luyten, and W. Daelemans (2017). Selecting relevant features from the electronic health record for clinical code prediction. Journal of Biomedical Informatics 74, 92-103. 
Scheurwegs, E., K. Luyckx, L. Luyten, W. Daelemans, and T. Van den Bulcke (2016). Data integration of structured and unstructured sources for assigning clinical codes to patient stays. Journal of the American Medical Informatics Association 23(e1), e11-e19.

Seijo-Pardo, B., I. Porto-Díaz, V. Bolón-Canedo, and A. Alonso-Betanzos (2017). Ensemble feature selection: Homogeneous and heterogeneous approaches. Knowledge-Based Systems 118, 124 - 139.

Somol, P. and J. Novovi (2010). Evaluating Stability and Comparing Output of Feature Selectors that Optimize Feature Subset Cardinality. IEEE Transactions on Pattern Analysis and Machine Intelligence 32(11), 1921-1939.

Wang, L., Y. Wang, and Q. Chang (2016). Feature selection methods for big data bioinformatics: A survey from the search perspective. Methods 111, $21-31$. Big Data Bioinformatics.

Witten I.H and Eibe Frank (2005). Data Mining: Practical machine learning tools and techniques. Morgan Kaufmann, 2005.

Yang, F. and K. Z. Mao (2011). Robust feature selection for microarray data based on multicriterion fusion. IEEE/ACM Transactions on Computational Biology and Bioinformatics (TCBB) 8(4), 1080-1092.

Yang, Y. H., Y. Xiao, and M. R. Segal (2004). Identifying differentially expressed genes from microarray experiments via statistic synthesis. Bioinformatics 21(7), 1084-1093.

Yeung, K. Y., R. E. Bumgarner, and A. E. Raftery (2005). Bayesian model averaging: develop- ment of an improved multi-class, gene selection and classification tool for microarray data. Bioinformatics 21(10), 2394-2402.

Zhao, Z., Liu H. (2007). Searching for interacting features, in: IJCAI 2007, vol. 7, 1156-1161. 
